\title{
Elimination of spiral waves in cardiac tissue by multiple electrical shocks
}

\author{
A. V. Panfilov \\ Department of Theoretical Biology, Utrecht University, Padualaan 8, Utrecht, 3584 CH, The Netherlands \\ Stefan C. Müller and Vladimir S. Zykov \\ Institut für Experimentelle Physik, Otto-von-Guericke-Universität, Universitätsplatz, 2, D-39106 Magdeburg, Germany \\ James P. Keener \\ Department of Mathematics, University of Utah, Salt Lake City, Utah 84112
}

(Received 17 March 1999; revised manuscript revised 28 September 1999)

\begin{abstract}
We study numerically the elimination of a spiral wave in cardiac tissue by application of multiple shocks of external current. To account for the effect of shocks we apply a recently developed theory for the interaction of the external current with cardiac tissue. We compare two possible feedback algorithms for timing of the shocks: a "local" feedback algorithm [1] (using an external electrode placed directly on the tissue) and a "global" feedback algorithm [2] (using the electrocardiogram). Our main results are: application of the external current causes a parametric resonant drift similar to that reported in previous model computations; the ratio of the threshold of elimination of the spiral wave by multiple shocks to the threshold of conventional single shock defibrillation in our model for cardiac tissue is about 0.5 , while earlier, less realistic models predicted the value about 0.2 ; we show that an important factor for successful defibrillation is the location of the feedback electrode and the best results are achieved if the feedback electrode or the ECG lead is located at the boundary (or edge) of the cardiac tissue; the "local" and the "global" feedback algorithms show similar efficiency.

PACS number(s): 87.19.Hh, 82.40.Fp, 05.45.-a
\end{abstract}

Many cardiac arrhythmias are characterized by rotating waves of excitation $[3,4]$ which are similar to spiral waves of excitation found in a wide variety of nonlinear excitable media [5]. Elimination of spiral waves in cardiac tissue is an important problem related to the treatment of potentially fatal cardiac arrhythmias.

The conventional method of elimination of spiral waves in cardiac tissue is defibrillation, which is achieved by the direct activation of most of the cardiac tissue by a single large electric shock. Although this protocol is often successful, it is desirable to reduce the necessary amount of current since large currents can cause tissue damage and are extremely painful. Recently, it has been suggested that spiral waves can be eliminated without overall activation of the tissue. One such approach uses overdrive local pacing of an arrhythmia [6]. Another approach exploits parametric resonant drift of spiral waves $[7,8]$. Here, it was shown that if certain properties of the medium are varied with the period of rotation of a spiral wave, the spiral wave will drift and can be eliminated at the boundary of the medium. The important feature of this method is that it does not require overall excitation of cardiac tissue and so can be achieved by smaller electric stimuli. Because electrical forcing can modify the period of spiral waves, determination of the resonant frequency may require a feedback loop control mechanism. Two possible algorithms for feedback have been proposed. The first algorithm requires placing an electrode directly on the cardiac tissue and changing the properties of the tissue at the moment the wave reaches the electrode $[1,9,10]$, or with some delay. The second possible algorithm is a "global feedback' control [2], and requires finding an integral char- acteristic of the distribution of excitation in the entire medium and forcing the medium on the basis of this information.

An important issue related to resonant drift is the means by which the tissue is stimulated. In all the papers listed above, the external stimulation was modeled by adding an extra transmembrane current directly to the equation describing the dynamics of the transmembrane potential. It is not clear how this (uniform) injection of transmembrane current can be achieved in real experiments. On the other hand, a natural way to change the instantaneous properties of cardiac tissue is by application of external electric current.

Recently homogenization theory has been used to show how externally applied currents are transformed into transmembrane currents [11-14]. This theory assumes that inhomogeneities, such as (but not limited to) gap junctional resistances, cause hyperpolarization and depolarization on the spatial scale of cells. Since spiral activity has a characteristic spatial scale of many cell lengths, it is permissible to "average" or "homogenize", these rapidly varying transmembrane currents and to determine an effective transmembrane current [11-14]. The goal of this paper is to examine the elimination of spiral waves via parametric resonant drift using this more realistic model of the interaction of external current with cardiac tissue. We study two types of feedback: one using a single electrode placed at the different locations directly on the cardiac tissue, similar to $[1,9,10]$, and the second, using the electrocardiogram measured at some distance from the tissue as an integral characteristic of the distribution of excitation in the tissue. For both feedback algorithms, we study how the location of the feedback device, 
delay in the feedback loop and the size of the tissue affect the threshold for elimination of reentrant waves.

\section{MODEL AND RESULTS}

We performed computations in a model of twodimensional cardiac tissue with transmembrane current described using simplified excitable dynamics of the FitzHughNagumo type, published earlier [15]

$$
\begin{gathered}
\partial e / \partial t=\nabla \cdot(\nabla e)-k e(e-a)(e-1)-e g+I_{s t}, \\
\partial g / \partial t=\left[\epsilon+\left(\mu_{1} g\right) /\left(\mu_{2}+e\right)\right][-g-k e(e-a-1)],
\end{gathered}
$$

where $e$ is the transmembrane potential, $-k e(e-a)(e-1)$ $-e g$ is the total transmembrane ionic current per unit area [15] and $I_{s t}$ is a stimulation current. This description reproduces many characteristics of cardiac tissue such as refractoriness, dispersion relation, rate-duration properties etc. The parameters of the model were $a=0.15, \mu_{1}=0.2 ; \mu_{2}=0.3, k$ $=8$, and $\epsilon=0.002$.

The action of external current on cardiac tissue was described using the homogenization theory. In Refs. [11-14] it was shown that averaging (integration) of the the transmembrane current over the rapidly varying spatial component of the transmembrane potential for the particular cubic righthand side function from Eq. (1), gives the following expression for $I_{s t}$ :

$$
I_{s t}=k * \frac{K * I_{e x}^{2}}{12}(1+a-3 e)
$$

where $I_{e x}$ is the external current and $K$ is some constant, which depends on the geometry of cells, the intracellular, extracellular and gap junctional resistances and does not depend on $I_{e x}$. (See Ref. [12] for details). Because we are interested only in relative threshold (compared to the defibrillation threshold, i.e., elimination with a single shock) the exact value of this coefficient $(K)$ is not important and we put it to $K=1$. To model shocks of external current, we use the expression for $I_{s t}$ from Eq. (2) with $I_{e x}$ some constant during the stimulus, and zero when the current is turned off. For these simulations, the duration of the shocks was about 5 times the duration of the action potential upstroke. By applying single stimuli of different strengths $I_{e x}$ we found that the threshold of conventional defibrillation was $I_{e x}=0.35$. We used this value as a reference to compare conventional defibrillation with the threshold values for the spiral wave elimination via resonant drift.

Equation (1) were numerically integrated on a square domain using the Euler method with Neumann boundary conditions with the dimensionless time step 0.07 and space step 0.6 . The dimensioned values of these steps were $0.28 \mathrm{~ms}$ and $0.6 \mathrm{~mm}$. The period of the spiral wave was $134 \mathrm{msec}$ with the upstroke of about $7.2 \mathrm{msec}$ and the wavelength $40 \mathrm{~mm}$. (The wavelength here is the product of the average period of a spiral wave times the speed of the wavefront). To calculate the electrocardiogram, we used the formula for the potential from [16]: $\Phi=\Sigma \partial e / \partial x_{i} \partial / \partial x_{i}(1 / R)$ where $\Sigma$ denotes the summation over all points of the numerical grid, $i=1,2,3$ is the index for the coordinate axes and $R$ is the distance from a lead to the point of the heart where $\partial e / \partial x_{i}$ is evaluated. We

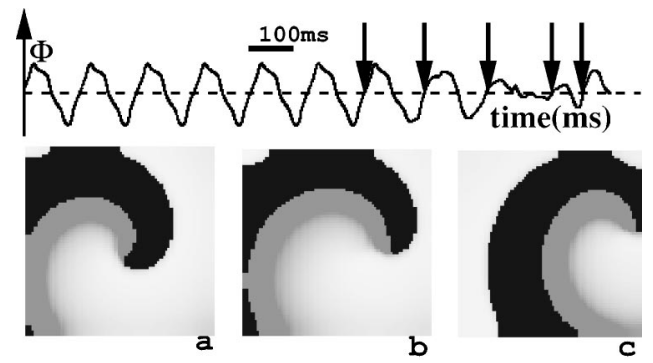

FIG. 1. The lower panel: elimination of the spiral wave due to the resonant drift in in the medium of the size $40 \mathrm{~mm} \times 40 \mathrm{~mm}$. (a) Pattern of excitation just before application of the first stimulus, (b) after $t=200 \mathrm{~ms}$, and (c) after $t=400 \mathrm{~ms}$. At $t=420 \mathrm{~ms}$ after beginning of the external stimulation the medium returns to rest. The relative amplitude of stimuli was 0.57 . The upper panel: ECG before and during stimulation. The location of the lead is at coordinates $(60,60,80 \mathrm{~mm})$ if the coordinates of the left lower corner were $(0,0,0)$. Moments of time when the stimuli were applied are marked by arrows.

assume the standard summation convention for the index $i$. A typical electrocardiogram from a spiral wave is shown in Fig. 1. Because the scale on the ECG is not relevant, no units are shown on the vertical axis in Fig. 1.

Figure 1 shows elimination of a spiral wave caused by the "global" feedback resonant drift. This procedure works as follows. We monitor the electrocardiogram produced by a rotating spiral. Because the electrocardiogram is an integral characteristic of the entire tissue, this is similar to the global feedback loop of [2]. We apply a stimulus with $I_{e x}=0.2$ each time the electrocardiogram traverses zero potential (arrows in Fig. 1) upward. Consequently the spiral wave gradually shifts to the right [Fig. 1(b)] and when the tip of the spiral wave collides with the boundary [Fig. 1(c)], the spiral wave disappears. In the case of Fig. 1, this occurred after 5 stimuli. The amplitude of the stimulus relative to the threshold of conventional defibrillation was $0.2 / 0.35=0.57$. Thus, the application of an external current caused parametric resonant drift of spiral waves similar to the drift observed under application of other periodic disturbances $[7,8,1]$.

Previous computations showed [1,2] that the resonant drift of spiral waves can be substantially affected by the phase shift in the feedback loop. Therefore, we studied the dependence of the threshold of elimination of a spiral wave as a function of this phase shift [Fig. 2(a), all lines except the lower gray thick line]. We did this for two feedback algorithms: "global" (using the ECG lead) and "local" (using an electrode placed directly on the tissue). For each of these two algorithms we performed computations for two feedback electrode locations: symmetrical and non-symmetrical. The black lines show the results for the "global" feedback algorithm, the gray lines show the results for the "local" feedback algorithm. In both cases delay was measured in relative units with delay 1 corresponding to the delay of $134 \mathrm{msec}$ (the period of rotation of a spiral wave in the undisturbed medium).

We see [Fig. 2(a)] that in all cases the minimal threshold is about $50 \%$ of the threshold of the conventional defibrillation. However, the threshold for symmetric locations (dashed lines) is on average higher, and for many values of the delay, the thresholds are quite high: around $75 \%$. 


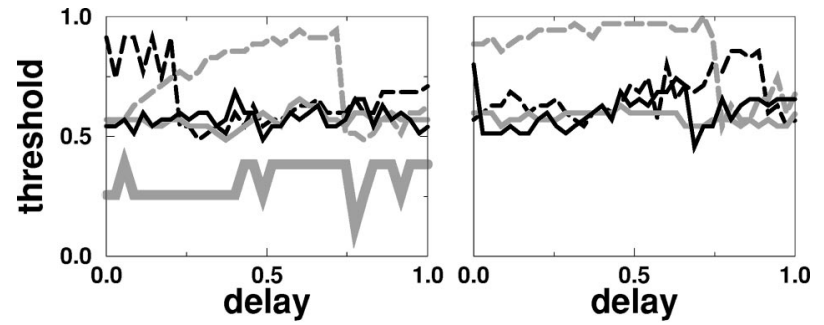

FIG. 2. Relative defibrillation threshold vs relative delay in the medium of the size: (a) $40 \mathrm{~mm} \times 40 \mathrm{~mm}$ and (b) $68 \times 68 \mathrm{~mm}$. The black solid lines show the results obtained for the "global" feedback loop via ECG signal from the lead at location $(60,60,80 \mathrm{~mm})$ in (a) and $(100,100,136 \mathrm{~mm})$ in (b), the dashed black lines show the same but for the lead located at $(20,20,80 \mathrm{~mm})$ in (a) and (34, $34,136 \mathrm{~mm}$ ) in (b); the solid grey lines show the "local" feedback via electrode located at $(1.2,1.2,0 \mathrm{~mm})$ in (a) and (b), and the dashed grey lines show the same but for the electrode located at $(20,20,0 \mathrm{~mm})$ for (a) and $(34,34,0 \mathrm{~mm})$ for (b). The thick grey line in Fig.a shows relative defibrillation threshold for forcing with a direct transmembrane current. Further explanations are in the text. The coordinates of the lower left corner are $(0,0,0)$. If the spiral wave was not eliminated after $4 s$ (about 30 stimuli) the defibrillation was considered as unsuccessful.

We did a similar computation for the medium of the $1.7 * 1.7$ larger size $68 \times 68 \mathrm{~mm}$ [Fig. 2(b)]. Although the results are similar to those from Fig. 2(a), there are some minor changes. First, the thresholds for the "global" feedback algorithm are lower, even with a symmetric location of the lead. On the other hand the thresholds for the "local" feedback algorithm with symmetric location of the electrode are higher and only in a small interval are they comparable to the thresholds from Fig. 2(a).

The obvious conclusions from these computations are that nonsymmetric locations are better than symmetric locations and both feedback algorithms are comparable. However, in our view, the "global" algorithm is more feasible for practical applications, as it does not require placing an electrode directly onto cardiac tissue.

With this in mind we made a more detailed study of the "global" feedback algorithm. Figure 3(a) shows the dependence of defibrillation thresholds for 4 different locations of a spiral wave. We see that the thresholds show similar dependences, independent of the initial conditions.

In previous computations we used a large number of

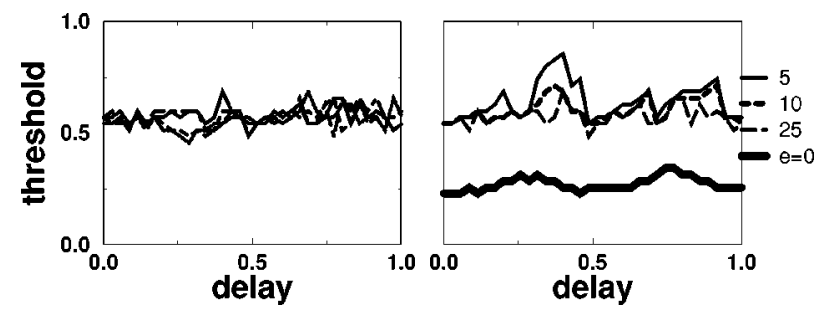

FIG. 3. Relative defibrillation threshold for the "global' feedback loop via ECG vs. relative delay in the feedback loop in the medium of the size $40 \mathrm{~mm} \times 40 \mathrm{~mm}$ for nonsymmetric lead position at $(60,60,80 \mathrm{~mm})$; (a) for four different initial locations of a spiral wave; (b) for different number of external stimuli (shown as numbers on the figure) and for fixed potential $e=0$ at the boundary and 30 external stimuli (the lower thick-black line). stimuli, up to 30 , to remove a spiral wave. We have studied effectiveness of our method if we reduce this maximal number of stimuli to 5,10 and 25 [Fig. 3(b)]. We see [Fig. 3(b)] that increasing the number of stimuli for some phase shifts decreases the threshold. However, even for 5 stimuli the "global" feedback algorithm gives reasonably good results: the average threshold is 0.63 and the minimal threshold of spiral elimination is almost the same. One might expect that increasing the number of stimuli should substantially decrease the minimal threshold. This is because the shift of a spiral is smaller for smaller stimulus amplitude and therefore one might expect that even very small stimuli can bring the spiral to the boundary and remove it if the stimulation time is sufficiently long. The fact that in our computation increasing of number of stimuli did not have a substantial effect on the elimination threshold might indicate that the limiting factor here is not bringing a spiral to the boundary, but the interaction of a spiral with the boundary. To check this suggestion, we did computations in which the potential at the boundary was fixed at $e=0$ [lower black thick line in Fig. 3(b)]. In this situation the relative threshold of spiral wave elimination is much lower than for no-flux boundary conditions which indicates that the limiting factor for spiral elimination in our case was the interaction of the spiral with the boundary. Note, however, that fixed potential boundary conditions are not physiologically realistic. More appropriate boundary conditions require use of the bidomain equations for cardiac tissue [20], which is beyond the scope of this paper.

Finally we compared the results obtained using our procedure of stimulation of cardiac tissue by pulses of the external current with the previously used procedure of forcing cardiac tissue with a direct transmembrane current $[1,9,10]$ [modeled by putting $I_{s t}$ in (1) to a constant value]. The lower gray thick line in Fig. 2(a) shows the results of our computations on this model. We see that for direct application of the transmembrane current the threshold is much lower than for application of the external current. It is typically about 0.25 , but for some delays it can be as low as 0.12 , which is about the value reported in Ref. [1]. These computations were made for the "local" feedback algorithm using an electrode located at the corner of the tissue of the size 40 $\times 40 \mathrm{~mm}$. Thus the relative thresholds for the feedback defibrillation found in this paper are about 2-3 times higher than reported in Refs. [1,9] and [10]. This fact has a simple explanation, since the effect of the external current in Eq. (2) is proportional to the square of the amplitude of stimulus, while putting $I_{s t}$ to constant in Eq. (1) is linear in $I_{s t}$.

\section{DISCUSSION}

In this paper, we studied the dependence of the elimination threshold as a function of the phase shift in the feedback loop (Fig. 2). The simulations show that in some cases (e.g., symmetric locations) such dependence is quite strong, however in other cases (non-symmetric locations) the dependence is rather flat. These results can be heuristically explained in the following way. With symmetric placement we find entrainment of spiral waves under forcing by pulses of external current. In particular, for some delays in the feedback loop the tip of the spiral wave has a stable complex- 
meandering trajectory with the center near the feedback electrode. Therefore the tip of a spiral wave never comes close to the boundary of the tissue and it cannot be eliminated by resonant drift. As a result, the thresholds for elimination of a spiral wave under external forcing comes close to the thresholds for conventional defibrillation. However at some delays, the tip trajectory expands, approaches the boundary, and elimination is possible. Such elimination is more pronounced in the smaller tissue, because the distance from the feedback electrode to the boundary there is smaller. This results in lower defibrillation thresholds for some delays in the feedback loop in the tissue of smaller size (compare the dashed gray lines in Figs. 2(a) and 2(b). If, however, the feedback electrode is located at the boundary, the entrainment trajectory always crosses the boundary and elimination of the spiral wave is determined mainly by the interaction of spiral wave with the boundary of the medium, which does not substantially depend on the phase shift in the feedback loop. As a consequence we have a flat dependence for non-symmetric cases in Fig. 2. A similar mechanism is at work for the different placements of the ECG electrode, however, because the ECG is an integral characteristic of the whole tissue, the effect is less pronounced. Such behavior of a spiral waves was first found in chemical experiments in a light sensitive $\mathrm{BZ}$ reaction and in numerical computations using the model for this reaction in Ref. [17] and later it was shown analytically in Refs. [18] and [19]. Here we show that this also occurs in the model of cardiac excitable tissue forced by pulses of external current.

The thresholds for feedback defibrillation are about half that of the threshold of the conventional defibrillation. However, because the energy is proportional to the square of the current, the damaging effects of the feedback defibrillation should be about one fourth that of conventional defibrillation.

In this study, we examined the elimination of a single rotating spiral wave as a model for tachycardia. We did not study elimination of ventricular fibrillation, which is believed to be associated with multiple spiral waves. We also did not take into account three-dimensional effects, cardiac anisotropy, the anatomy of the heart, etc. Their effects on defibrillation can be significant and should be studied in the future.

\section{ACKNOWLEDGMENT}

We acknowledge the useful remarks of Dr. V. N. Biktashev in the preparation of this paper.
[1] V. N. Biktashev and A. V. Holden, J. Theor. Biol. 169, 101 (1994).

[2] V. S. Zykov, A. S. Mikhailov, and S. C. Müller, Phys. Rev. Lett. 78, 3398 (1997).

[3] M. A. Allessie, F. I. M. Bonke, and F. J. G. Schopman, Circ. Res. 33, 54 (1973).

[4] J. M. Davidenko et al., Nature (London) 355, 349 (1991).

[5] A. T. Winfree and S. H. Strogatz, Nature (London) 311, 611 (1984).

[6] M. J. Osborn, in Cardiology: Fundamentals and Practice. 2nd ed., edited by E. R. Giuliani et al. (Mosby-Year Book Inc., St. Louis, 1991), pp. 1080-1113.

[7] K. I. Agladze, V. A. Davydov, and A. S. Mikhailov, Pis'ma Zh. Éksp. Teor. Fiz. 45, 601 (1987) [JETP Lett. 45, 767 (1987)].

[8] O. Steibock, V. S. Zykov, and S. C. Müller, Nature (London) 366, 322 (1993).
[9] V. N. Biktashev and A. V. Holden, Phys. Rev. B 260, 211 (1995).

[10] V. N. Biktashev and A. V. Holden, Phys. Rev. B 263, 1373 (1996).

[11] A. Pumir and V. I. Krinsky, Physica D 91, 205 (1996).

[12] J. P. Keener, J. Theor. Biol. 178, 313 (1996).

[13] J. P. Keener and A. V. Panfilov, Biophys. J. 71, 1335 (1996).

[14] A. Pumir and V. I. Krinsky, J. Theor. Biol. 185, 189 (1997).

[15] R. R. Aliev and A. V. Panfilov, Chaos, Solitons and Fractals 7, 293 (1996)

[16] J. A. Abildskov and R. L. Lux, J. Cardiovasc. Electrophysiol. 2, 224 (1991).

[17] S. Grill, V. S. Zykov, and S. C. Müller, Phys. Rev. Lett. 75, 3368 (1995).

[18] E. V. Nikolaev, V. N. Biktashev, and A. V. Holden, Chaos, Solitons and Fractals 9, 363 (1998).

[19] A. Karma and V. Zykov, Phys. Rev. Lett. 83, 2453 (1999).

[20] C. S. Henriques, Crit. Rev. Biomed. Eng. 21, 1 (1993). 